\title{
Freeness of conic-line arrangements in $\mathbb{P}^{2}$
}

\author{
Hal Schenck*and Ştefan O. Tohăneanu
}

\begin{abstract}
Let $\ell=\bigcup_{i=1}^{n} C_{i} \subseteq \mathbb{P}^{2}$ be a collection of smooth rational plane curves. We prove that the addition-deletion operation used in the study of hyperplane arrangements has an extension which works for a large class of arrangements of smooth rational curves, giving an inductive tool for understanding the freeness of the module $\Omega^{1}(\mathcal{C})$ of logarithmic differential forms with pole along $\ell$. We also show that the analog of Terao's conjecture (freeness of $\Omega^{1}(\mathcal{C}$ ) is combinatorially determined if $\mathcal{C}$ is a union of lines) is false in this setting.
\end{abstract}

Mathematics Subject Classification (2000). Primary 52B30; Secondary 14J60.

Keywords. Line arrangement, curve arrangement, module of derivations.

\section{Introduction}

One of the fundamental objects associated to a hyperplane arrangement $\mathcal{A} \subseteq \mathbb{P}_{\mathbb{K}}(V)$ is the module $\Omega^{1}(\mathcal{A})$ of logarithmic one-forms with pole along the arrangement or (dually) the module $D(\mathcal{A})$ of derivations tangent to the arrangement. Both are graded $S=\operatorname{Sym}\left(V^{*}\right)$ modules; $D(\mathcal{A}) \subseteq \operatorname{Der}_{\mathbb{K}}(S)$ is defined via:

Definition 1.1. $D(\mathcal{A})=\left\{\theta \mid \theta\left(l_{i}\right) \in\left\langle l_{i}\right\rangle\right.$ for all $l_{i}$ such that $\left.V\left(l_{i}\right) \in \mathcal{A}\right\}$.

Over a field of characteristic zero, $D(\mathcal{A}) \simeq E \oplus D_{0}(\mathcal{A})$, where $E$ is the Euler derivation and $D_{0}(\mathcal{A})$ corresponds to the module of syzygies on the Jacobian ideal of the defining polynomial of $\mathcal{A}$. When $\mathbb{K}=\mathbb{C}$ or $\mathbb{R}$, an elegant theorem of Terao relates the freeness of the module $D(\mathcal{A})$ to the Poincaré polynomial of $V \backslash \mathcal{A}$. In this note, we restrict to $\mathbb{P}^{2}$, but broaden the class of curves which make up the arrangement. In particular, suppose

$$
e=\bigcup_{i=1}^{n} C_{i},
$$

where each $C_{i}$ is a smooth rational plane curve; call such a collection a conic-line (CL) arrangement.

\footnotetext{
${ }^{*}$ Schenck was supported by NSF 03-11142, 07-07667, NSA 904-03-1-0006.
} 
Example 1.2. For the CL arrangement below, $D(\mathcal{C}) \simeq S(-1) \oplus S(-2) \oplus S(-5)$.

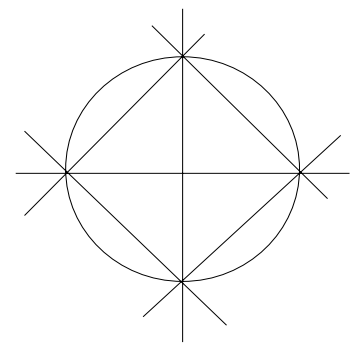

1.1. Line arrangements. We begin with some facts about hyperplane arrangements; for more information see Orlik and Terao [5]. A hyperplane arrangement $\mathcal{A}$ is a finite collection of codimension one linear subspaces of a fixed vector space $V$. A is central if each hyperplane contains the origin of $V$. The intersection lattice $L_{\mathcal{A}}$ of $\mathcal{A}$ consists of the intersections of the elements of $\mathcal{A}$; the rank of $x \in L_{\mathcal{A}}$ is simply the codimension of $x . V$ is the lattice element $\hat{0}$; the rank one elements are the hyperplanes themselves. $\mathcal{A}$ is called essential if $\operatorname{rank} L_{\mathcal{A}}=\operatorname{dim} V$.

Definition 1.3. The Möbius function $\mu: L_{\mathcal{A}} \rightarrow \mathbb{Z}$ is defined by

$$
\begin{aligned}
& \mu(\hat{0})=1, \\
& \mu(t)=-\sum_{s<t} \mu(s), \quad \text { if } \hat{0}<t .
\end{aligned}
$$

We now restrict to the case that $V$ is complex. A foundational result is that the Poincaré polynomial of $X=V \backslash \mathcal{A}$ is purely combinatorial; in particular

$$
P(X, t)=\sum_{x \in L_{\mathcal{A}}} \mu(x) \cdot(-t)^{\operatorname{rank}(x)} .
$$

An arrangement $\mathcal{A}$ is free if $D(\mathcal{A}) \simeq \oplus S\left(-a_{i}\right)$; the $a_{i}$ are called the exponents of $\mathcal{A}$. Terao's famous theorem [11] states that if $D(\mathcal{A}) \simeq \oplus S\left(-a_{i}\right)$, then $P(X, t)=$ $\prod\left(1+a_{i} t\right)$. If $\mathcal{A} \subseteq \mathbb{C}^{3}$ is central, then $\mathcal{A}$ also defines a set of lines in $\mathbb{P}^{2}$, and obviously $X=\mathbb{C}^{3} \backslash \mathcal{A} \simeq \mathbb{C}^{*} \times \tilde{X}$, where $\tilde{X}$ is the complement of the corresponding arrangement of lines in $\mathbb{P}^{2}$. Hence

$$
P(\tilde{X}, t)=1+(n-1) t+\left(\sum_{\substack{x \in L_{\mathcal{A}} \\ \operatorname{rank}(x)=2}} \mu(x)-n+1\right) t^{2}
$$

It follows from Terao's theorem that if $D_{0}(\mathcal{A}) \simeq S(-a) \oplus S(-b)$, then $P(\tilde{X}, t)=$ $(1+a t)(1+b t)$. This can be generalized [9] to line arrangements which are not free, using the Chern polynomial. The motivating question of this paper is: what happens if the arrangement of lines is replaced with a CL arrangement? 
1.2. Rational curve arrangements. In [2], Cogolludo-Agustín studies the complement of an arrangement of rational curves in $\mathbb{P}^{2}$, where the individual curves can have singularities, and can meet non-transversally. The main result is that the cohomology ring of the complement to a rational curve arrangement is generated by logarithmic 1and 2-forms and its structure depends on a finite number of invariants of the curve. One fact is that if $\tilde{X}$ is the complement of an arrangement of $n$ irreducible curves in $\mathbb{P}^{2}$, then

$$
\begin{gathered}
h^{1}(\tilde{X}, \mathbb{C})=n-1, \\
h^{2}(\tilde{X}, \mathbb{C})=1+\sum_{p \in \operatorname{Sing}(\mathcal{C})}\left(r_{p}-1\right)-\sum_{1}^{n}\left(\chi\left(\hat{C}_{i}\right)-1\right),
\end{gathered}
$$

where $r_{p}$ is the number of branches passing through $p$, and $\hat{C}_{i}$ is the normalization of $C_{i}$. Since we are assuming that all the $C_{i}$ are smooth and rational, we have that

$$
h^{2}(\tilde{X}, \mathbb{C})=\sum_{p \in L_{2}(\ell)}\left(r_{p}-1\right)-|\mathcal{E}|+1,
$$

where the intersection poset $L(\mathcal{C})$ is defined precisely as for a linear arrangement (typically, $L(\mathcal{C})$ is only a poset, not a lattice).

1.3. Milnor and Tjurina numbers. A crucial distinction between line and curve arrangements, even in our simple setting, is the difference between the Milnor and Tjurina numbers at a singularity. Let $C=V(f)$ be a reduced (but not necessarily irreducible) curve in $\mathbb{C}^{2}$, let $(0,0) \in C$, and let $\mathbb{C}\{x, y\}$ denote the ring of convergent power series.

Definition 1.4. The Milnor number of $C$ at $(0,0)$ is

$$
\mu_{(0,0)}(C)=\operatorname{dim}_{\mathbb{C}} \mathbb{C}\{x, y\} /\left\langle\frac{\partial f}{\partial x}, \frac{\partial f}{\partial y}\right\rangle .
$$

To define $\mu_{p}$ for an arbitrary point $p$, we translate so that $p$ is the origin.

Definition 1.5. The Tjurina number of $C$ at $(0,0)$ is

$$
\tau_{(0,0)}(C)=\operatorname{dim}_{\mathbb{C}} \mathbb{C}\{x, y\} /\left\langle\frac{\partial f}{\partial x}, \frac{\partial f}{\partial y}, f\right\rangle .
$$

Definition 1.6. A singularity is quasihomogeneous iff there exists a holomorphic change of variables so the defining equation becomes weighted homogeneous; $f(x, y)=\sum c_{i j} x^{i} y^{j}$ is weighted homogeneous if there exist rational numbers $\alpha, \beta$ such that $\sum c_{i j} x^{i \cdot \alpha} y^{j \cdot \beta}$ is homogeneous. 
In [6], Reiffen proved that if $f(x, y)$ is a convergent power series with isolated singularity at the origin, then $f(x, y)$ is in the ideal generated by the partial derivatives if and only if $f$ is quasihomogeneous (see [8] for a generalization).

As noted earlier, for a line arrangement with defining polynomial $F, D_{0}(\mathcal{A})$ consists of the syzygies on the Jacobian ideal $J_{F}$ of $F$. If $V(F) \subseteq \mathbb{P}^{2}$ is a reduced curve, then after a change of coordinates, we may assume that $V(F)$ has no singularities on the line $z=0$. Dehomogenizing so that $f(x, y)=F(X, Y, 1)$ yields

$$
\operatorname{deg}\left(J_{F}\right)=\operatorname{dim}_{\mathbb{C}} \mathbb{C}[x, y] /\left\langle\frac{\partial f}{\partial x}, \frac{\partial f}{\partial y}, f\right\rangle=\sum_{p \in \operatorname{Sing}(C)} \tau_{p}(f) .
$$

It follows that if all the singular points are quasihomogeneous, then

$$
\operatorname{deg}\left(J_{F}\right)=\sum_{p \in \operatorname{Sing}(V(f))} \mu_{p}(f) .
$$

For a line arrangement, the singularities are always quasihomogeneous, but this is not the case for CL arrangements:

Example 1.7. Let $\mathcal{C}=V\left(x y(x-y)(x-2 y)\left(x^{2}-x z+y^{2}-y z\right)\right)$ be as below:

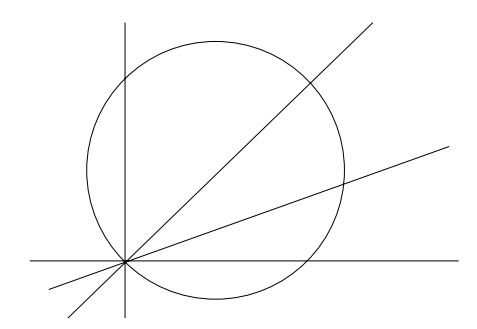

$C$ has five singular points, all ordinary. When $p$ is an ordinary singularity and $C$ has $n$ distinct branches at $p$, then $\mu_{p}(C)=(n-1)^{2}$, so the sum of the Milnor numbers is 20 . However, $\operatorname{deg}(J)=19$; at $(0: 0: 1)$ we have $\mu=16$ but $\tau=15$.

1.4. Criteria for freeness. The first criterion for the freeness of $D(\mathcal{A})$ is the following:

Proposition 1.8 (Saito, [7]). A is free exactly when there exist $n+1$ elements

$$
\theta_{i}=\sum_{j=0}^{n} f_{i j} \frac{\partial}{\partial x_{j}} \in D(\mathcal{A})
$$

such that the determinant of the matrix $\left[f_{i j}\right]$ is a nonzero constant multiple of the defining polynomial of $\mathcal{A}$. 
Saito's criterion holds for an arrangement of reduced hypersurfaces $\ell \subseteq \mathbb{P}^{n}$; let $e=V(F)$ where $F=f_{1} \ldots f_{d}$, and $\operatorname{gcd}\left(f_{i}, f_{j}\right)=1$. By induction,

$$
\theta\left(f_{1} \ldots f_{d}\right)=f_{1} \theta\left(f_{2} \ldots f_{d}\right)+f_{2} \ldots f_{d} \theta\left(f_{1}\right) \in\left\langle f_{1} \ldots f_{d}\right\rangle,
$$

so we have

$$
\begin{aligned}
D(\mathcal{C}) & =\left\{\theta \in \operatorname{Der}_{\mathbb{K}}(S) \mid \theta\left(f_{i}\right) \in\left\langle f_{i}\right\rangle, i=1, \ldots, d\right\} \\
& =\left\{\theta \in \operatorname{Der}_{\mathbb{K}}(S) \mid \theta(F) \in\langle F\rangle\right\} .
\end{aligned}
$$

Any arrangement of (reduced) hypersurfaces will have a singular locus of codimension two. As for a linear arrangement, $D(\mathcal{C}) \simeq E \oplus D_{0}(\mathcal{C})$, with $D_{0}(\mathcal{C})=\operatorname{syz}\left(J_{F}\right)$, so freeness is equivalent to $\operatorname{pdim}\left(S / J_{F}\right)=2$ (so also equivalent to $J_{F}$ CohenMacaulay). By the Hilbert-Burch theorem ([3]), any codimension two CohenMacaulay ideal $I$ with $m+1$ generators is generated by the maximal minors of an $m \times(m+1)$ matrix $M$, whose columns generate the module of first syzygies on $I$. So when $I=J_{F}$, appending a column vector $\left[x_{0}, \ldots, x_{n}\right]$ to $M$ and taking the determinant yields a multiple of $F$, by Euler's formula. Saito's criterion is most useful when an explicit set of candidates for the generating set of $\operatorname{syz}\left(J_{F}\right)$ is known.

There are two other fundamental tools that can be used to prove that a line arrangement is free. The first method is based on an inductive operation known as deletion-restriction: given an arrangement $\mathcal{A}$ and a choice of hyperplane $H \in \mathcal{A}$, set

$$
\mathcal{A}^{\prime}=\mathcal{A} \backslash H \text { and } \quad \mathcal{A}^{\prime \prime}=\left.\mathcal{A}\right|_{H} .
$$

The collection $\left(\mathcal{A}^{\prime}, \mathcal{A}, \mathcal{A}^{\prime \prime}\right)$ is called a triple, and a triple yields (see Proposition 4.45 of [5]) a left exact sequence

$$
0 \longrightarrow D\left(\mathcal{A}^{\prime}\right)(-1) \stackrel{\cdot H}{\longrightarrow} D(\mathscr{A}) \longrightarrow D\left(\mathscr{A}^{\prime \prime}\right) .
$$

For a triple with $\mathcal{A} \subseteq \mathbb{P}^{2}$, more is true (see [10]): after pruning the Euler derivations and sheafifying, there is an exact sequence

$$
0 \longrightarrow \mathscr{D}_{0}{ }^{\prime}(-1) \longrightarrow \mathscr{D}_{0} \longrightarrow i_{*} \mathscr{D}_{0}{ }^{\prime \prime} \longrightarrow 0,
$$

where $i: H \hookrightarrow \mathbb{P}^{2} ; i_{*} \mathscr{D}_{0}{ }^{\prime \prime} \simeq \mathcal{O}_{H}\left(1-\left|\mathcal{A}^{\prime \prime}\right|\right)$. In [12], Terao showed that freeness of a triple is related via:

Theorem 1.9 (Addition-Deletion). Let $\left(\mathcal{A}^{\prime}, \mathcal{A}, \mathcal{A}^{\prime \prime}\right)$ be a triple. Then any two of the following imply the third:

- $D(\mathcal{A}) \simeq \bigoplus_{i=1}^{n} S\left(-b_{i}\right)$;

- $D\left(\mathcal{A}^{\prime}\right) \simeq S\left(-b_{n}+1\right) \bigoplus_{i=1}^{n-1} S\left(-b_{i}\right)$;

- $D\left(\mathcal{A}^{\prime \prime}\right) \simeq \bigoplus_{i=1}^{n-1} S\left(-b_{i}\right)$.

Theorem 1.9 applies in general, not just to arrangements in $\mathbb{P}^{2}$. A smooth conic is intrinsically a $\mathbb{P}^{1}$, so it is natural to ask if $\mathrm{CL}$ arrangements which admit a short 
exact sequence similar to (1) have an addition-deletion theorem; we tackle this in the next two sections.

A second criterion for freeness is special to the case of line arrangements; to state it we need to define freeness for multiarrangements. A multiarrangement $(\mathcal{A}, \boldsymbol{m})$ is an arrangement together with a multiplicity $m_{i}$ for each hyperplane. The module of derivations consists of $\theta$ such that $l_{i}^{m_{i}} \mid \theta\left(l_{i}\right)$. As shown by Ziegler in [15], freeness of multiarrangements is not combinatorial; for recent progress see [13].

Theorem 1.10 (Yoshinaga's multiarrangement criterion, [16]). $\mathcal{A} \subseteq \mathbb{P}^{2}$ is free iff $\pi(\mathcal{A}, t)=(1+t)(1+a t)(1+b t)$ and for all $H \in \mathcal{A}$ the multiarrangement $\left.\mathcal{A}\right|_{H}$ has minimal generators in degree $a$ and $b$.

The main results of this paper (Theorems 2.5 and 3.4) show that an additiondeletion construction holds for CL arrangements with quasihomogeneous singularities; the freeness of Example 1.2 is explained by our results. As one application, we show that a free CL arrangement, when restricted to different lines, can yield multiarrangements with different exponents; hence any version of Theorem 1.10 for $\mathrm{CL}$ arrangements will be quite subtle. An addition-deletion theorem for multiarrangements has recently been proven by Abe-Terao-Wakefield in [1]; our results are the first (to our knowledge) to give an inductive criterion for freeness for nonlinear arrangements.

\section{Addition-deletion for a line}

Let $\left(\ell^{\prime}, \mathcal{C}, \ell^{\prime \prime}\right)$ be a triple of CL arrangements in $\mathbb{P}^{2}$, where $\ell^{\prime}=\ell \backslash\{L\}, \ell^{\prime \prime}=\ell_{L}$ and $L \in \mathcal{C}$ is a line. We begin by examining some examples:

Example 2.1. Let $\ell^{\prime}$ be the union of

$$
\begin{aligned}
& C_{1}=x^{2}-x z+5 y^{2}-5 y z=0, \\
& C_{2}=x^{2}+2 y^{2}-x z-2 y z=0, \\
& L_{1}=x=0, \\
& L_{2}=y=0, \\
& L_{3}=x+y-z=0 .
\end{aligned}
$$

$D\left(\mathcal{C}^{\prime}\right)$ is free with exponents $\{1,2,4\}$, and the degree of the Jacobian ideal is 28 , which is equal to the sum of the Milnor numbers at the intersection points. Therefore at each singular point $\tau=\mu$. If we restrict to any line, the corresponding multiarrangement has two points of multiplicity 3 , and it follows from [13] that the exponents are $\{3,3\}$. Hence the obvious generalization of Yoshinaga's criterion does not hold. 


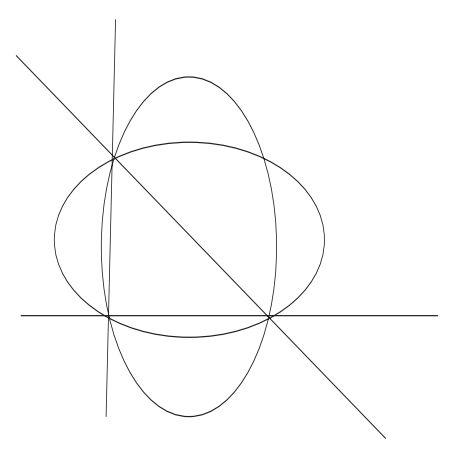

Example 2.2. Let $L_{4}=\{x-y=0\}$ and let $\ell_{1}=\ell^{\prime} \cup L_{4}$. The degree of the Jacobian ideal is 39 , which is equal to the sum of Milnor numbers at the points. It will follow from our results that $D\left(\ell_{1}\right)$ is free with exponents $\{1,2,5\}$.

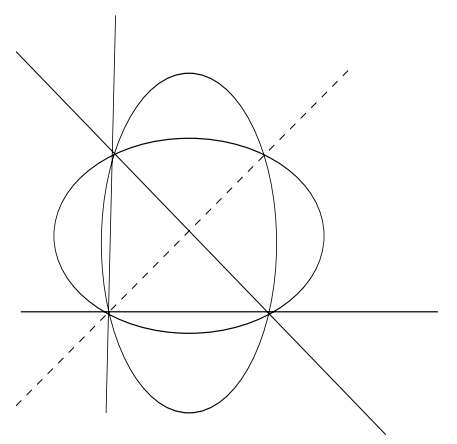

Example 2.3. Let $L_{4}=\{x-2 y=0\}$ and let $\ell_{2}=\ell^{\prime} \cup L_{4}$. Then $\ell_{2}$ is free with exponents $\{1,3,4\}$. The degree of the Jacobian ideal is 37 , whereas the sum of the Milnor numbers is 38; the singularity at $(0: 0: 1)$ has $\tau=15$ and $\mu=16$.

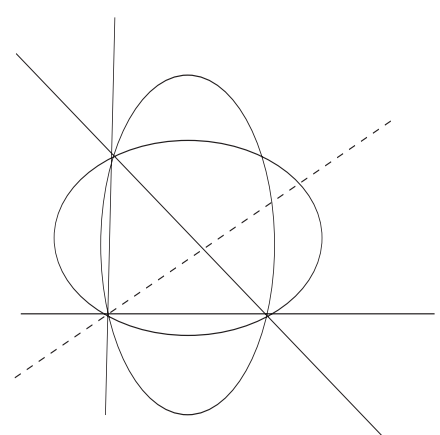


For $\mathrm{CL}$ arrangements similar to $\mathscr{C}_{1}$, there is an addition-deletion theorem:

Definition 2.4. A triple $\left(\mathcal{C}^{\prime}, \mathcal{C}, \mathcal{C}^{\prime \prime}\right)$ of $\mathrm{CL}$ arrangements is called quasihomogeneous if $\tau=\mu$ at each singular point of $\ell^{\prime}$ and $\ell^{\text {. }}$.

Theorem 2.5. Let $\left(\mathcal{C}^{\prime}, \mathcal{C}, \mathcal{C}^{\prime \prime}\right)$ be a quasihomogeneous triple with $|L \cap \mathcal{C}|=$ $\left|\zeta^{\prime \prime}\right|=k$. The following are equivalent:

(1) $e^{\prime}$ is free with exponents $\{1, k-1, a\}$.

(2) $\mathcal{C}$ is free with exponents $\{1, k-1, a+1\}$.

Examples 2.1 and 2.2 illustrate the theorem; before giving the proof of Theorem 2.5 , we need some preliminaries.

Lemma 2.6. Let $L=\{x=0\}$. Then the maps $p: D\left(\mathcal{C}^{\prime}\right) \rightarrow D(\mathcal{C}), p(\theta)=x \theta$ and $q: D(\mathcal{C}) \rightarrow D\left(\mathcal{C}^{\prime \prime}\right), q\left(a \partial_{x}+b \partial_{y}+c \partial_{z}\right)=b(0, y, z) \partial_{y}+c(0, y, z) \partial_{z}$ are well defined and yield an exact sequence

$$
0 \longrightarrow D\left(e^{\prime}\right) \longrightarrow D(\mathcal{C}) \longrightarrow D\left(\mathcal{C}^{\prime \prime}\right) .
$$

Proof. Let $f=x f^{\prime}$ be the defining polynomial of $\ell$, where $f^{\prime}$ is the defining polynomial of $\ell^{\prime}$. Then the defining polynomial of $\ell^{\prime \prime}$ is $f^{\prime \prime}=\operatorname{Rad}\left(\left.f^{\prime}\right|_{x=0}\right)$. If $\theta^{\prime} \in D\left(\mathcal{C}^{\prime}\right)$, then $\theta^{\prime}\left(f^{\prime}\right)=P f^{\prime}$ for some $P \in S$;

$$
p\left(\theta^{\prime}\right)(f)=x \theta^{\prime}\left(x f^{\prime}\right)=x\left(f^{\prime} \theta^{\prime}(x)+x \theta^{\prime}\left(f^{\prime}\right)\right) \in\langle f\rangle .
$$

So $p$ is well defined and injective. Let $\theta=a \partial_{x}+b \partial_{y}+c \partial_{z} \in D(\mathcal{C})$. Then $\theta(x)=$ $a \in\langle x\rangle$, so $a=x a^{\prime}$. If $\theta \in \operatorname{ker}(q)$, then $b=x b^{\prime}$ and $c=x c^{\prime}$, hence $\theta=x \theta^{\prime}$, where $\theta^{\prime}=a^{\prime} \partial_{x}+b^{\prime} \partial_{y}+c^{\prime} \partial_{z}$. Because $\theta \in D(\mathcal{C}), \theta\left(f^{\prime}\right)=x \theta^{\prime}\left(f^{\prime}\right) \in\left\langle f^{\prime}\right\rangle$. Since $x$ and $f^{\prime}$ are relatively prime, we get that $\theta^{\prime}\left(f^{\prime}\right) \in\left\langle f^{\prime}\right\rangle$, which implies that $\theta \in \operatorname{Im}(p)$.

It remains to show is that $q$ is well defined. For suitable $u_{i}, v_{i} \in \mathbb{C}$ and $m_{i} \in \mathbb{Z}$ we have that

$$
\left.f^{\prime}\right|_{x=0}=\prod_{i}\left(u_{i} y+v_{i} z\right)^{m_{i}}, \text { so } f^{\prime \prime}=\prod_{i}\left(u_{i} y+v_{i} z\right) .
$$

Let $L^{\prime}$ be a line in $\mathcal{C}^{\prime}$ defined by the vanishing of $t_{i} x+u_{i} y+v_{i} z=0$ for some $i$ and $t_{i} \in \mathbb{C}$, and let $\theta=a \partial_{x}+b \partial_{y}+c \partial_{z} \in D(\mathcal{C})$. Then $\theta\left(L^{\prime}\right) \in\left\langle L^{\prime}\right\rangle$, so evaluating at $x=0$ and using the earlier observation that $a=x a^{\prime}$, we find $\left(b(0, y, z) \partial_{y}+c(0, y, z) \partial_{z}\right)\left(u_{i} y+v_{i} z\right) \in\left\langle u_{i} y+v_{i} z\right\rangle$.

Now suppose $C$ is a conic in $\ell^{\prime}$; after a change of coordinates we may assume $C$ intersects $L=\{x=0\}$ in the points $(0: 0: 1)$ and $(0: u: v)$. Then 
$C=x A+y(v y-u z)$ and $\left.C\right|_{x=0}=y(v y-u z)$, where $A$ is some linear form. We have

$$
\begin{aligned}
\theta(C)=a & \left(A+x \partial_{x}(A)\right)+x\left(b \partial_{y}(A)+c \partial_{z}(A)\right) \\
& +b \partial_{y}(y(v y-u z))+c \partial_{z}(y(v y-u z)) \in\langle C\rangle
\end{aligned}
$$

Evaluating at $x=0$ and again using that $a=x a^{\prime}$ we find

$$
\left(b(0, y, z) \partial_{y}+c(0, y, z) \partial_{z}\right)(y(v y-u z)) \in\langle y(v y-u z)\rangle .
$$

Since $y$ and $v y-u z$ are relatively prime we obtain

$$
\begin{aligned}
\left(b(0, y, z) \partial_{y}+c(0, y, z) \partial_{z}\right)(y) & \in\langle y\rangle, \\
\left(b(0, y, z) \partial_{y}+c(0, y, z) \partial_{z}\right)(v y-u z) & \in\langle v y-u z\rangle .
\end{aligned}
$$

This shows that for each factor $u_{i} y+v_{i} z$ of $f^{\prime \prime}$,

$$
\left(b(0, y, z) \partial_{y}+c(0, y, z) \partial_{z}\right)\left(u_{i} y+v_{i} z\right) \in\left\langle u_{i} y+v_{i} z\right\rangle,
$$

so the map $q$ is well defined. It follows that $D_{0}\left(\mathcal{C}^{\prime \prime}\right)=\mathbb{C}[y, z](-(k-1))$, where $k=\left|L \cap \ell^{\prime}\right|=\operatorname{deg}\left(f^{\prime \prime}\right)$. A similar argument works if $C$ is tangent to $L$.

Lemma 2.7. Let $X$ and $Y$ be two reduced plane curves with no common component, meeting at a point $p$. Then

$$
\mu_{p}(X \cup Y)=\mu_{p}(X)+\mu_{p}(Y)+2(X \cdot Y)_{p}-1,
$$

where $(X \cdot Y)_{p}$ is the intersection number of $X$ and $Y$ at $p$.

Proof. See [14], Theorem 6.5.1; the point is that the Milnor fiber is a connected curve, and the result follows from using the additivity of the Euler characteristic and the interpretation of $\mu_{p}$ as the first Betti number of the Milnor fiber.

Proposition 2.8. Let $\left(\mathcal{C}^{\prime}, \mathcal{C}, \mathcal{C}^{\prime \prime}\right)$ be a quasihomogeneous triple. Then

$$
0 \longrightarrow \mathcal{D}_{0}{ }^{\prime}(-1) \longrightarrow \mathcal{D}_{0} \longrightarrow i_{*} \mathscr{D}_{0}{ }^{\prime \prime}
$$

is also right exact.

Proof. It follows from Lemma 2.6 that quotienting by the Euler derivation and sheafifying yields the left exact sequence above; so it will suffice to show that $H P\left(D_{0}, t\right)=H P\left(D_{0}^{\prime}(-1), t\right)+H P\left(i_{*} D_{0}^{\prime \prime}, t\right)$, where $H P(-, t)$ denotes the Hilbert polynomial. For a CL arrangement $\mathcal{C}$ with $m$ lines and $n$ conics, let $d=2 n+m-1$. We have an exact sequence:

$$
0 \longrightarrow D_{0}(\mathcal{C}) \longrightarrow S^{3} \longrightarrow S(d) \longrightarrow S(d) / J \longrightarrow 0,
$$


where $S=\mathbb{K}[x, y, z]$ and $J$ is the Jacobian ideal of the defining polynomial of $\mathcal{C}$. Since

$$
\begin{gathered}
H P\left(D_{0}, t\right)=3\left(\begin{array}{c}
t+2 \\
2
\end{array}\right)-\left(\begin{array}{c}
t+2+d \\
2
\end{array}\right)+\operatorname{deg}(J), \\
H P\left(D_{0}^{\prime}(-1), t\right)=3\left(\begin{array}{c}
t+1 \\
2
\end{array}\right)-\left(\begin{array}{c}
t+d \\
2
\end{array}\right)+\operatorname{deg}\left(J^{\prime}\right),
\end{gathered}
$$

we find that

$$
H P\left(D_{0}, t\right)-H P\left(D_{0}^{\prime}(-1), t\right)=\operatorname{deg}(J)-\operatorname{deg}\left(J^{\prime}\right)+t-2 d+2 .
$$

By the assumption that $\left(\mathcal{C}^{\prime}, \mathcal{C}, \mathcal{C}^{\prime \prime}\right)$ is a quasihomogeneous triple,

$$
\operatorname{deg}(J)=\sum_{p \in \operatorname{Sing}(C)} \mu_{p}(C) \text { and } \operatorname{deg}\left(J^{\prime}\right)=\sum_{p \in \operatorname{Sing}\left(C^{\prime}\right)} \mu_{p}\left(C^{\prime}\right)
$$

Let $\alpha$ be the sum of Milnor numbers of points off $L$, so

$$
\operatorname{deg}(J)=\alpha+\sum_{p \in L \cap C^{\prime}} \mu_{p}(C)
$$

Since $\mu_{p}(L)=0$, by Lemma 2.7 , the above is

$$
\alpha+\sum_{p \in L \cap C^{\prime}}\left(\mu_{p}\left(C^{\prime}\right)+2\left(L \cdot C^{\prime}\right)_{p}-1\right)
$$

As $\operatorname{deg}\left(J^{\prime}\right)=\alpha+\sum_{p \in L \cap C^{\prime}} \mu_{p}\left(C^{\prime}\right)$ and $\left|L \cap C^{\prime}\right|=k$, we obtain

$$
\operatorname{deg}(J)-\operatorname{deg}\left(J^{\prime}\right)=2 \sum_{p \in L \cap C^{\prime}}\left(L \cdot C^{\prime}\right)_{p}-k .
$$

By Bezout's theorem,

$$
\sum_{p \in L \cap C^{\prime}}\left(L \cdot C^{\prime}\right)=d,
$$

so $\operatorname{deg}(J)-\operatorname{deg}\left(J^{\prime}\right)=2 d-k$, hence

$$
H P\left(D_{0}, t\right)-H P\left(D_{0}^{\prime}(-1), t\right)=t+2-k=t+1-\left(\left|\mathcal{C}^{\prime \prime}\right|-1\right) .
$$

Since $i_{*} \mathscr{D}_{0}{ }^{\prime \prime}=\mathcal{O}_{L}\left(1-\left|\mathcal{C}^{\prime \prime}\right|\right)$, this yields the result.

Definition 2.9. A coherent sheaf $\mathcal{F}$ on $\mathbb{P}^{r}$ is $m$-regular iff $H^{i} \mathcal{F}(m-i)=0$ for every $i \geq 1$. The smallest number $m$ such that $\mathscr{F}$ is $m$-regular is denoted by $\operatorname{reg}(\mathcal{F})$. 
Lemma 2.10. For a quasihomogeneous triple with $\left|\mathcal{C}^{\prime \prime}\right|=k$,

$$
\operatorname{reg}\left(\mathscr{D}_{0}\right) \leq \max \left\{\operatorname{reg}\left(\mathscr{D}_{0}^{\prime}\right)+1, k-1\right\} .
$$

Proof. Immediate from Proposition 2.8 (see [10]).

Lemma 2.11. If $\mathfrak{D}_{0}^{\prime}=\mathcal{O}_{\mathbb{P}^{2}}(1-k) \oplus \mathcal{O}_{\mathbb{P}^{2}}(-a)$, then there is an exact sequence of $S$-modules

$$
0 \longrightarrow D_{0}^{\prime}(-1) \longrightarrow D_{0} \longrightarrow D_{0}^{\prime \prime} \longrightarrow 0 .
$$

Proof. For all $t, H^{1}\left(\mathscr{D}_{0}^{\prime}(t-1)\right)=0$, so the long exact sequence in cohomology arising from Proposition 2.8 and the vanishing of $H^{1}\left(\mathscr{D}_{0}^{\prime}(t)\right)$ yield an exact sequence:

$$
0 \longrightarrow \bigoplus_{t} H^{0}\left(\mathscr{D}_{0}^{\prime}(-1)(t)\right) \longrightarrow \bigoplus_{t} H^{0}\left(\mathscr{D}_{0}(t)\right) \longrightarrow \bigoplus_{t} H^{0}\left(\mathscr{D}_{0}^{\prime \prime}(t)\right) \longrightarrow 0
$$

Theorem A.4.1 of [3] relates a graded module to its sheaf and local cohomology (at the maximal ideal $\mathfrak{m}$ ) modules:

$$
0 \longrightarrow H_{\mathfrak{m}}^{0}\left(D_{0}\right) \longrightarrow D_{0} \longrightarrow \bigoplus_{t} H^{0}\left(D_{0}(t)\right) \longrightarrow H_{\mathfrak{m}}^{1}\left(D_{0}\right) \longrightarrow 0 .
$$

This is true also for $D_{0}^{\prime}(-1)$ and $D_{0}^{\prime \prime}$. By [3], A.4.3, $H_{\mathfrak{m}}^{0}(M)=H_{\mathfrak{m}}^{1}(M)=0$ if $\operatorname{depth}(M) \geq 2$. Lemma 2.1 of [4] gives the desired bound on depth for the modules of derivations, which concludes the proof.

The next two lemmas prove the two implications (1) $\Rightarrow(2)$ and (2) $\Rightarrow(1)$ of Theorem 2.5. In what follows, $\left(\mathcal{C}^{\prime}, \mathcal{C}, \mathcal{C}^{\prime \prime}\right)$ is a quasihomogeneous triple, with $L$ a line and $|L \cap \mathcal{\ell}|=\left|\mathcal{\ell}^{\prime \prime}\right|=k$.

Lemma 2.12. If $\mathcal{C}^{\prime}$ is free with $\exp \left(\mathcal{C}^{\prime}\right)=\{1, k-1, a\}$, then $C$ is free with $\exp (\mathcal{C})=\{1, k-1, a+1\}$.

Proof. First, if $S=\mathbb{K}\left[x_{1}, \ldots, x_{\ell}\right]$, and $S(-i)$ is a free graded $S$-module with generator in degree $i$, then the Hilbert series satisfies

$$
H S(S(-i), t)=\frac{t^{i}}{(1-t)^{\ell}} .
$$

If $\mathcal{C}^{\prime}$ is free with exponents $\{1, k-1, a\}$, then $D_{0}^{\prime}(-1) \simeq S(-k) \oplus S(-1-a)$. It follows from the proof of Proposition 2.8 that $H S\left(D_{0}^{\prime \prime}, t\right)=\frac{t^{k-1}}{(1-t)^{2}}$. So by Lemma 2.11 and the additivity of Hilbert series on an exact sequence,

$$
H S\left(D_{0}\right)=\frac{t^{k}+t^{a+1}}{(1-t)^{3}}+\frac{t^{k-1}}{(1-t)^{2}}=\frac{t^{a+1}+t^{k-1}}{(1-t)^{3}} .
$$


Since $D_{0}^{\prime} \simeq S(-k+1) \oplus S(-a), \operatorname{reg}\left(D_{0}^{\prime}\right)=\max \{k-1, a\}$. By Lemma 2.10 , if $a \geq k-1$, then $\operatorname{reg}\left(D_{0}\right) \leq a+1$; and if $a \leq k-2$, then $\operatorname{reg}\left(D_{0}\right) \leq k$. If $a \leq k-2$, then a free resolution for $D_{0}$ is of the form

$$
0 \longleftarrow D_{0} \longleftarrow S(-k+1) \oplus S(-a-1) \oplus S(-b)^{d} \longleftarrow S(-b)^{d} \longleftarrow 0 .
$$

From regularity constraints, $b$ must be at most $k$. As this is a minimal free resolution, and it is impossible to have a syzygy on a single generator, the only situation which can actually arise occurs when $b=k$ :

$$
0 \longleftarrow D_{0} \longleftarrow S(-k+1) \oplus S(-a-1) \oplus S(-k)^{d} \longleftarrow S(-k)^{d} \longleftarrow 0 .
$$

Let $t_{1}, t_{2}$ be two independent derivations in $D_{0}$ of degrees $\operatorname{deg}\left(t_{1}\right)=a+1$ and $\operatorname{deg}\left(t_{2}\right)=k-1$; our computation of the Hilbert series, combined with the fact that $\operatorname{pdim}\left(D_{0}\right) \leq 1$ means such derivations must exist. Let $E, t_{1}^{\prime}, t_{2}^{\prime}$ be a basis for $D^{\prime}$ with $\operatorname{deg}\left(t_{1}^{\prime}\right)=a$ and $\operatorname{deg}\left(t_{2}^{\prime}\right)=k-1$, and $E$ the Euler derivation.

Now note that $t_{1}^{\prime} \in D_{0}^{\prime} \backslash D_{0}$, for otherwise in $D_{0}$ there would be an element of degree $a$. So $t_{1}^{\prime}(x) \notin\langle x\rangle$. Since $D \subset D^{\prime}$, then $t_{1}=f_{1} E+x t_{1}^{\prime}$ and $t_{2}=$ $f_{2} E+u t_{2}^{\prime}+f t_{1}^{\prime}$, where $u$ is a constant, $\operatorname{deg}(f)=k-1-a, \operatorname{deg}\left(f_{1}\right)=a$ and $\operatorname{deg}\left(f_{2}\right)=k-2$. For a resolution as above, $g t_{1}=L t_{2}$, where $L$ is a linear form and $\operatorname{deg}(g)=k-(a+1)$. Hence

$$
\left(g f_{1}-L f_{2}\right) E+(g x-L f) t_{1}^{\prime}+(-L u) t_{2}^{\prime}=0,
$$

and since $E, t_{1}^{\prime}$ and $t_{2}^{\prime}$ form a basis, we find that $u$ vanishes and $g x=L f$. But $\left(t_{2}-f_{2} E\right)(x) \in\langle x\rangle$ and $t_{1}^{\prime}(x) \notin\langle x\rangle$. Since $u=0, x$ must divide $f$, and so $g=L g^{\prime}$ for some $g^{\prime}$. Since $g t_{1}=L t_{2}$, we obtain $t_{2}=g^{\prime} t_{1}$, a contradiction. If $a \geq k-1$, simply switch the roles of $a$ and $k$ above.

Lemma 2.13. If $\mathcal{C}$ is free with $\exp (\mathcal{C})=\{1, k-1, a+1\}$, then $\mathcal{C}^{\prime}$ is free with $\exp \left(\mathcal{C}^{\prime}\right)=\{1, k-1, a\}$.

Proof. In order to obtain an appropriate vanishing, we need to dualize. Apply $\operatorname{Hom}\left(-, \mathcal{O}_{\mathbb{P}^{2}}\right)$ to the exact sequence

$$
0 \longrightarrow \mathscr{D}_{0}{ }^{\prime}(-1) \longrightarrow \mathscr{D}_{0} \longrightarrow i_{*}{D_{0}}^{\prime \prime} \longrightarrow 0 .
$$

The vanishing of $\operatorname{Hom}_{\mathcal{O}_{\mathbb{P}^{2}}}\left(\mathcal{O}_{\mathbb{P}^{1}}(t), \mathcal{O}_{\mathbb{P}^{2}}\right)$ and $\operatorname{Ext}_{\mathcal{O}_{\mathbb{P}^{2}}}^{1}\left(D_{0}, \mathcal{O}_{\mathbb{P}^{2}}\right)$ yields an exact sequence

$$
0 \longrightarrow \mathscr{D}_{0}^{\vee} \longrightarrow \mathscr{D}_{0}^{\prime \vee}(1) \longrightarrow \operatorname{Ext}_{S}^{1}\left(\mathcal{O}_{L}(1-k), \mathcal{O}_{\mathbb{P}^{2}}\right) \longrightarrow 0 .
$$

The free $\mathcal{O}_{\mathbb{P}^{2}}$ resolution for $\mathcal{O}_{L}(1-k)$ is

$$
0 \longrightarrow O_{\mathbb{P}^{2}}(-k) \longrightarrow O_{\mathbb{P}^{2}}(1-k) \longrightarrow \mathcal{O}_{L}(1-k) \longrightarrow 0,
$$


so $\operatorname{Ext}_{S}^{1}\left(\mathcal{O}_{L}(1-k), \mathcal{O}_{\mathbb{P}^{2}}\right) \simeq \mathcal{O}_{L}(k)$. Since $D_{0}^{\vee}=\mathcal{O}_{\mathbb{P}^{2}}(k-1) \oplus \mathcal{O}_{\mathbb{P}^{2}}(a+1)$, combining this with the long exact sequence in cohomology yields a regularity bound

$$
\operatorname{reg}\left(\mathscr{D}_{0}^{\prime \vee}\right) \leq \max \left\{\operatorname{reg}\left(\mathscr{D}_{0}^{\vee}\right)+1,1-k\right\},
$$

and the exact sequence of $S$-modules

$$
0 \longrightarrow D_{0}^{\vee}(-1) \longrightarrow D_{0}^{\prime \vee} \longrightarrow S(k-1) / L \longrightarrow 0
$$

with $D_{0}^{\vee}=S(k-1) \oplus S(a+1)$. So

$$
H S\left(D_{0}^{\prime \vee}\right)=\frac{t^{-a}+t^{1-k}}{(1-t)^{3}} .
$$

An argument as in the proof of Lemma 2.12 shows that $D_{0}^{\prime \vee}=S(a) \oplus S(k-1)$, hence $D\left(\ell^{\prime}\right)$ is free with exponents $\{1, k-1, a\}$.

Corollary 2.14. A free CL arrangement, when restricted to a line, can yield different multiarrangements.

Proof. In Example 2.2, add the line $L=\{x-\alpha y+(\alpha-1) z=0\}$, where $\alpha \notin$ $\{0,1,-5,-2, \infty\}$. Then $L$ passes through $(1: 1: 1)$, and the choices for $\alpha$ ensure that $L$ is not tangent to any conic, and misses all singularities save $(1: 1: 1)$. The new arrangement is quasihomogeneous, and $L$ meets $\mathscr{C}_{1}$ in six points. By Theorem 2.5, the new arrangement is free with exponents $\{1,3,5\}$.

Restrict this new arrangement to the line $L_{3}=\{x+y-z=0\}$. After a change of coordinates, we obtain a multiarrangement with defining polynomial

$$
x^{3} y^{3}(x-y)(\alpha x-y) .
$$

This is exactly Ziegler's example from [15]: $\alpha=-1$ gives exponents $\{3,5\}$, and for $\alpha \neq-1$, the exponents are $\{4,4\}$.

\section{Addition-deletion for a conic}

Let $\left(\ell^{\prime}, \mathcal{C}, \ell^{\prime \prime}\right)$ be a triple of CL arrangements in $\mathbb{P}^{2}$, where $C$ is a conic in $\ell$, and $e^{\prime}=\ell \backslash\{C\}, \ell^{\prime \prime}=\left.\ell^{\prime}\right|_{C}$. We begin with some examples.

Example 3.1. Suppose $\ell$ is as in Example 2.2, so $\ell$ has quasihomogeneous singularities, and is free with exponents $\{1,2,5\}$. If we delete one of the conics, the resulting arrangement $\ell^{\prime}$ is free and quasihomogeneous, with exponents $\{1,2,3\}$.

When $k$ is odd, the situation is more complicated: 
Example 3.2. Let $\mathcal{C}^{\prime}$ be the braid arrangement $A_{3}=V(x y z(x-z)(y-z)(x+y-z))$, and $\ell=\ell^{\prime} \cup C$, where the conic $C=V(x y+7 x z+13 y z)$. $\ell^{\prime}$ is a free arrangement with exponents $\{1,2,3\}$, and $\left|\mathcal{C}^{\prime \prime}\right|=7$. $\mathcal{C}$ is also quasihomogeneous, but not free.

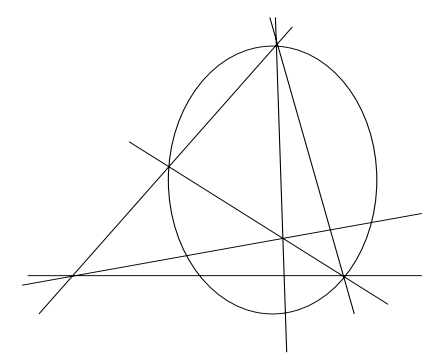

Example 3.3. Let $C$ be the quasihomogeneous CL arrangement with defining polynomial $\left(x^{2}-x z+2 y^{2}-2 y z\right) x y(x+y-z)$. $D(\mathcal{C})$ is free with exponents $\{1,2,2\}$. Deleting the conic yields a free line arrangement with exponents $\{1,1,1\}$.

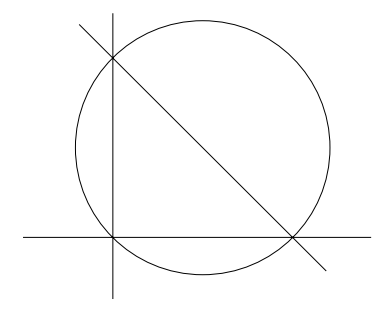

Theorem 3.4. Let $\left(\mathcal{C}^{\prime}, \mathcal{C}, \mathcal{C}^{\prime \prime}\right)$ be a quasihomogeneous triple, with $\left|C \cap \mathcal{C}^{\prime}\right|=$ $\left|e^{\prime \prime}\right|=k$. If $k=2 m$ then the following are equivalent:

(1) $\ell^{\prime}$ is free with $\exp \left(\mathcal{C}^{\prime}\right)=\{1, m, a\}$;

(2) $\mathcal{C}$ is free with $\exp (\mathcal{C})=\{1, m, a+2\}$.

If $k=2 m+1$ then:

(1) $\exp \left(\mathcal{C}^{\prime}\right)=\{1, m, m\} \Longleftrightarrow \exp (\mathcal{C})=\{1, m+1, m+1\}$;

(2) if $\exp \left(\mathcal{C}^{\prime}\right)=\{1, m, a\}$ with $a \neq m$ then $\mathcal{C}$ is not free;

(3) if $\exp (\mathcal{C})=\{1, m+1, a+1\}$ with $a \neq m$ then $\mathcal{C}^{\prime}$ is not free.

We begin with some preliminaries. After an appropriate change of coordinates, we may suppose that $C=\left\{y^{2}-x z=0\right\}$. Let $i$ be the composition of the maps

$$
\mathbb{P}^{1} \stackrel{v}{\longrightarrow} C \hookrightarrow \mathbb{P}^{2},
$$


where $v(s: t)=\left(s^{2}: s t: t^{2}\right)$, and let $\psi$ be the composite map

$$
S=\mathbb{K}[x, y, z] \stackrel{\phi}{\longrightarrow} \mathbb{K}\left[s^{2}, s t, t^{2}\right] \hookrightarrow \mathbb{K}[s, t],
$$

where $\phi(x)=s^{2}, \phi(y)=s t, \phi(z)=t^{2}$.

Let $\theta=a_{1} \partial_{x}+a_{2} \partial_{y}+a_{3} \partial_{z} \in D(\mathcal{C})$ be a derivation. Then $\theta(C) \in\langle C\rangle$, which means $-z a_{1}+2 y a_{2}-x a_{3}=\left(y^{2}-x z\right) P$ for some $P \in S$. Via the map $\psi$ this translates into

$$
t^{2} \psi\left(a_{1}\right)-2 s t \psi\left(a_{2}\right)+s^{2} \psi\left(a_{3}\right)=0 .
$$

So there exist $Q_{1}, Q_{2} \in \mathbb{K}[s, t]$ such that

$$
\begin{aligned}
& \psi\left(a_{1}\right)=s Q_{1}, \\
& \psi\left(a_{2}\right)=\frac{t Q_{1}+s Q_{2}}{2}, \\
& \psi\left(a_{3}\right)=t Q_{2} .
\end{aligned}
$$

If $\psi: S \rightarrow A$ is a ring map and $M$ is an $A$-module, let $M_{\psi}$ denote the $S$-module obtained by restriction of scalars.

Proposition 3.5. There is an exact sequence of $S$-modules

$$
0 \longrightarrow D\left(\mathcal{C}^{\prime}\right)(-2) \stackrel{\cdot C}{\longrightarrow} D(\mathcal{C}) \stackrel{\rho}{\longrightarrow} D\left(\mathcal{A}^{\prime \prime}\right)_{\psi},
$$

where

$$
\rho\left(a_{1} \partial_{x}+a_{2} \partial_{y}+a_{3} \partial_{z}\right)=Q_{1} \partial_{s}+Q_{2} \partial_{t},
$$

for every $a_{1} \partial_{x}+a_{2} \partial_{y}+a_{3} \partial_{z} \in D(\mathcal{C})$ and $Q_{1}, Q_{2}$ are defined as above; and $\mathcal{A}^{\prime \prime}$ is the arrangement of the reduced points $i^{-1}\left(C \cap \mathcal{C}^{\prime}\right)$ in $\mathbb{P}^{1}$.

Proof. It is easy to check that $\rho$ is a homomorphism. For exactness, note:

$$
\begin{aligned}
\theta=a_{1} \partial_{x}+a_{2} \partial_{y}+a_{3} \partial_{z} \in \operatorname{ker}(\rho) & \Longleftrightarrow Q_{1}=0 \text { and } Q_{2}=0 \\
& \Longleftrightarrow \psi\left(a_{1}\right)=\psi\left(a_{2}\right)=\psi\left(a_{3}\right)=0 \\
& \Longleftrightarrow a_{1}, a_{2}, a_{3} \in\left\langle y^{2}-x z\right\rangle \\
& \Longleftrightarrow \theta=C \theta^{\prime} \text { with } \theta^{\prime} \in D\left(\iota^{\prime}\right) .
\end{aligned}
$$

It remains to show that the image of $\rho$ is in $D\left(\mathcal{A}^{\prime \prime}\right)_{\psi}$. Suppose $\alpha x+\beta y+\gamma z=0$ is a line of $\mathcal{C}$. Let $\theta=a_{1} \partial_{x}+a_{2} \partial_{y}+a_{3} \partial_{z} \in D(\mathcal{C})$. Then

$$
\alpha a_{1}+\beta a_{2}+\gamma a_{3}=(\alpha x+\beta y+\gamma z) P_{1}
$$


for some $P_{1} \in S$. Therefore

$$
\alpha \psi\left(a_{1}\right)+\beta \psi\left(a_{2}\right)+\gamma \psi\left(a_{3}\right)=\left(\alpha s^{2}+\beta s t+\gamma t^{2}\right) \psi\left(P_{1}\right),
$$

which implies

$$
(2 \alpha s+\beta t) Q_{1}+(\beta s+2 \gamma t) Q_{2}=2\left(\alpha s^{2}+\beta s t+\gamma t^{2}\right) \psi\left(P_{1}\right) .
$$

This means that $\left(Q_{1} \partial_{s}+Q_{2} \partial_{t}\right)\left(\alpha s^{2}+\beta s t+\gamma t^{2}\right) \in\left(\alpha s^{2}+\beta s t+\gamma t^{2}\right) \mathbb{K}[s, t]$. Since $\alpha s^{2}+\beta s t+\gamma t^{2}$ is the defining polynomial of the two points $i^{-1}(\{\alpha x+\beta y+$ $\gamma z=0\} \cap C)$ in $\mathbb{P}^{1}$, we get that $Q_{1} \partial_{s}+Q_{2} \partial_{t}$ is a derivation on the arrangement of these two points.

Suppose $C^{\prime}=\left\{u_{0} x^{2}+u_{1} x y+u_{2} x z+u_{3} y^{2}+u_{4} y z+u_{5} z^{2}=0\right\}$ is a conic in the CL arrangement $\mathcal{C}$. Let $\theta=a_{1} \partial_{x}+a_{2} \partial_{y}+a_{3} \partial_{z} \in D(\mathcal{C})$. Computations as above show that

$$
\begin{gathered}
\left(Q_{1} \partial_{s}+Q_{2} \partial_{t}\right)\left(u_{0} s^{4}+u_{1} s^{3} t+\left(u_{2}+u_{3}\right) s^{2} t^{2}+u_{4} s t^{3}+u_{5} t^{4}\right) \\
\in\left(u_{0} s^{4}+u_{1} s^{3} t+\left(u_{2}+u_{3}\right) s^{2} t^{2}+u_{4} s t^{3}+u_{5} t^{4}\right) \mathbb{K}[s, t]
\end{gathered}
$$

Since $u_{0} s^{4}+u_{1} s^{3} t+\left(u_{2}+u_{3}\right) s^{2} t^{2}+u_{4} s t^{3}+u_{5} t^{4}$ is the defining polynomial of the four points $i^{-1}\left(C^{\prime} \cap C\right)$ in $\mathbb{P}^{1}$, we get that $Q_{1} \partial_{s}+Q_{2} \partial_{t}$ is a derivation on the arrangement of these four points. Similar arguments work in the case of tangencies.

Let $\theta=a_{1} \partial_{x}+a_{2} \partial_{y}+a_{3} \partial_{z} \in D(\mathcal{C})_{d}$ such that $\rho(\theta)=s \partial_{s}+t \partial_{t}$. Then $a_{1}, a_{2}, a_{3} \in S_{d}$ with $\psi\left(a_{1}\right)=s^{2}, \psi\left(a_{2}\right)=s t, \psi\left(a_{3}\right)=t^{2}$. Thus $d=1$ and $\theta$ is the Euler derivation in $D(\mathcal{C})$. So quotienting by the Euler derivations yields an exact sequence:

$$
0 \longrightarrow D_{0}^{\prime}(-2) \stackrel{\cdot C}{\longrightarrow} D_{0} \stackrel{\rho}{\longrightarrow}\left(D\left(\mathscr{A}^{\prime \prime}\right)_{0}\right)_{\psi} .
$$

Since $\left|\mathcal{A}^{\prime \prime}\right|=k$, after sheafifying, $\mathscr{D}_{0}\left(\mathcal{A}^{\prime \prime}\right)=\mathcal{O}_{\mathbb{P}^{1}}(-k)$, and hence the sheafification of $D_{0}\left(\mathcal{A}^{\prime \prime}\right)_{\psi}$ is $i_{*} \mathcal{O}_{\mathbb{P}^{1}}(-k)$.

Lemma 3.6. $H P\left(i_{*} \mathcal{O}_{\mathbb{P}^{1}}(-k), t\right)=2 t+1-k$.

Proof. Case 1: $k=2 m$. Let $E$ be the divisor of the reduced $k$ points $i^{-1}\left(C \cap \complement^{\prime}\right)$. Then the ideal sheaf $d_{E}=\langle f\rangle$, where $f \in \mathbb{K}[s, t]$ of degree $k=2 m$. There exists $g \in S_{m}$, unique modulo $\left(y^{2}-x z\right)$, such that $g\left(s^{2}, s t, t^{2}\right)=f$. Clearly $y^{2}-x z$ cannot divide $g$, otherwise $g\left(s^{2}, s t, t^{2}\right)=0=f$, so the ideal of the reduced $k$ points on $C$ is $\left\langle y^{2}-x z, g\right\rangle$. Hence $i_{*} d_{E}=\langle\bar{g}\rangle$ as an ideal of $S /\left\langle y^{2}-x z\right\rangle$. As an $S$-module, it has free resolution

$$
0 \longrightarrow S(-2-m) \stackrel{\cdot C}{\longrightarrow} S(-m) \longrightarrow\langle\bar{g}\rangle \longrightarrow 0,
$$


which yields

$$
H P\left(i_{*} \mathcal{O}_{\mathbb{P}^{1}}(-2 m), t\right)=\left(\begin{array}{c}
t+2-m \\
2
\end{array}\right)-\left(\begin{array}{c}
t-m \\
2
\end{array}\right)=2 t+1-2 m .
$$

Case 2: $k=2 m+1$. Let $E$ be the divisor of the reduced $k$ points $i^{-1}\left(C \cap \complement^{\prime}\right)$. Then the ideal sheaf $d_{E}=\langle f\rangle$, where $f \in K[s, t]$ of degree $k=2 m+1$. Let $L_{1}, L_{2} \in \mathbb{K}[s, t]_{1}$ be two independent linear forms which do not divide $f$, and let $f_{i}=L_{i} f$. Since $\left\langle L_{1} f, L_{2} f\right\rangle=\left\langle L_{1}, L_{2}\right\rangle \cap\langle f\rangle$, then $\left\langle f_{1}, f_{2}\right\rangle$ defines the same ideal sheaf on $\mathbb{P}^{1}$ as $\langle f\rangle$. So $d_{E}=\left\langle f_{1}, f_{2}\right\rangle$.

Both $f_{1}$ and $f_{2}$ are of even degree $2 m+2$. So there exist $g_{1}, g_{2} \in S=\mathbb{K}[x, y, z]$ of degree $m+1$ such that $g_{i}\left(s^{2}, s t, t^{2}\right)=f_{i}, i=1,2$. Next we show that $J=$ $\left\langle y^{2}-x z, g_{1}, g_{2}\right\rangle$ is the ideal of the reduced points $C \cap \mathcal{C}^{\prime}$ on $C$. To see this, note that if $p \in C \cap \mathcal{C}^{\prime}$, then $f_{i}\left(i^{-1}(p)\right)=0, i=1,2$. So $g_{i}(p)=0, i=1,2$, and hence $g_{i} \in J, i=1,2$. Clearly $y^{2}-x z$ does not divide $g_{i}$, otherwise $f_{i}$ is identically zero. Also, suppose $g_{2}=\lambda g_{1}+P\left(y^{2}-x z\right)$, where $\lambda$ is a constant. Then $f_{2}=\lambda f_{1}$, i.e. $L_{2}=\lambda L_{1}$; a contradiction. So $J$ is the ideal of $2 m+1$ points on the conic $y^{2}-x z=0$. By the Hilbert-Burch theorem, such an ideal is minimally generated by the $2 \times 2$ minors of

$$
\left[\begin{array}{ll}
x & y \\
y & z \\
\alpha & \beta
\end{array}\right]
$$

where both $\alpha$ and $\beta$ have degree $m$. So indeed $\left\langle y^{2}-x z, g_{1}, g_{2}\right\rangle=J$, and $i_{*} d_{E}=$ $\left\langle\overline{g_{1}}, \overline{g_{2}}\right\rangle \subseteq S /\left\langle y^{2}-x z\right\rangle$. As an $S$-module it has free resolution

$$
0 \longrightarrow S^{2}(-2-m) \stackrel{\left[\begin{array}{l}
x \\
y \\
y
\end{array}\right]}{\longrightarrow} S^{2}(-1-m) \longrightarrow\left\langle\overline{g_{1}}, \overline{g_{2}}\right\rangle \longrightarrow 0
$$

so for the odd case we find that

$$
\begin{aligned}
H P\left(i_{*} \mathcal{O}_{\mathbb{P}^{1}}(-2 m-1), t\right) & =2\left(\begin{array}{c}
t+1-m \\
2
\end{array}\right)-2\left(\begin{array}{c}
t-m \\
2
\end{array}\right) \\
& =2 t-2 m=2 t+1-(2 m+1) .
\end{aligned}
$$

Proposition 3.7. For a quasihomogeneous triple $\left(\ell^{\prime}, \mathcal{C}, \mathcal{C}^{\prime \prime}=\left.\ell^{\prime}\right|_{C}\right)$, the sequence

$$
0 \longrightarrow \mathscr{D}_{0}^{\prime}(-2) \stackrel{\cdot C}{\longrightarrow} D_{0} \longrightarrow i_{*} \mathcal{O}_{L}(-k) \longrightarrow 0
$$

is exact, where $i: L \stackrel{\left[s^{2}: s t: t^{2}\right]}{\longrightarrow} \mathbb{P}^{2}$. 
Proof. We have $H P\left(D_{0}, t\right)-H P\left(D_{0}^{\prime}(-2), t\right)=2 t-4 d+9+\left(\operatorname{deg}\left(J_{f}\right)-\operatorname{deg}\left(J_{f^{\prime}}\right)\right)$, where $d+1$ is the degree of the defining polynomial $f$ of $\mathcal{C}$ and $f^{\prime}$ is the defining polynomial of $\ell^{\prime}$. Since $\left(\ell^{\prime}, \mathcal{C}, \mathcal{C}^{\prime \prime}\right)$ is a quasihomogeneous triple, Bezout's theorem and Lemma 2.7 imply that $\operatorname{deg}\left(J_{f}\right)-\operatorname{deg}\left(J_{f^{\prime}}\right)=4 d-4-k$ and hence

$$
H P\left(D_{0}, t\right)-H P\left(D_{0}^{\prime}(-2), t\right)=2 t+1-k .
$$

By Lemma 3.6, this is exactly the Hilbert polynomial of the sheaf $i_{*} \mathcal{O}_{\mathbb{P}^{1}}(-k)$ associated to $D_{0}\left(\zeta^{\prime \prime}\right)_{\psi}$.

Lemma 3.8. For a quasihomogeneous triple such that $\mathcal{C}^{\prime}$ is free with exponents $\{1, m, a\}$,

$$
0 \longrightarrow D_{0}^{\prime}(-2) \longrightarrow D_{0} \longrightarrow D_{0}\left(\mathcal{A}^{\prime \prime}\right)_{\psi} \longrightarrow 0
$$

is exact.

Proof. As we have seen,

$$
\bigoplus_{t} H^{0}\left(\left(i_{*} \mathcal{O}_{L}(-k)\right)(t)\right)=\bigoplus_{t} H^{0}\left(\mathcal{O}_{\mathbb{P}^{1}}(2 t-k)\right)=D_{0}\left(\mathcal{A}^{\prime \prime}\right)_{\psi}
$$

With the assumption on $\ell^{\prime}, H^{1}\left(\mathscr{D}_{0}^{\prime}(t-2)\right)$ vanishes for all $t$, and exactness follows as in the proof of Lemma 2.11 .

Theorem 3.4 will follow from the next two lemmas.

Lemma 3.9. Let $\left(\mathcal{C}^{\prime}, \mathcal{C}, \mathcal{C}^{\prime \prime}\right)$ be a quasihomogeneous triple, with $\left|C \cap \ell^{\prime}\right|=$ $\left|\mathcal{C}^{\prime \prime}\right|=k$. If $\mathcal{C}^{\prime}$ is free with exponents $\{1, m, a\}$, then the following holds.

(1) If $k=2 m$ then $\mathcal{C}$ is free with $\exp (\mathcal{C})=\{1, m, a+2\}$.

(2) If $k=2 m+1$ and $a=m$, then $\mathcal{C}$ is free with $\exp (\mathcal{C})=\{1, m+1, m+1\}$.

(3) If $k=2 m+1$ and $a \neq m$, then $C$ is not free.

Proof. It follows from the computations in the proof of Lemma 3.6 that

- if $k=2 m+1$, then $H S\left(D_{0}\left(\mathcal{A}^{\prime \prime}\right)_{\psi}, t\right)=\frac{2 t^{m+1}}{(1-t)^{2}}$;

- if $k=2 m$, then $H S\left(D_{0}\left(\mathcal{A}^{\prime \prime}\right)_{\psi}, t\right)=\frac{t^{m}(1+t)}{(1-t)^{2}}$.

Combining these results yields the Hilbert series of $D_{0}$.

Case 1: $k=2 m$. By Lemma 3.8,

$$
H S\left(D_{0}, t\right)=\frac{t^{m}+t^{a+2}}{(1-t)^{3}}
$$

Since $\operatorname{pdim}\left(D_{0}\right) \leq 1$, this means that there exist minimal generators $\theta, \eta \in D_{0}$ with $\operatorname{deg}(\theta)=m$ and $\operatorname{deg}(\eta)=a+2$. Suppose $\left\{E, \theta_{1}, \theta_{2}\right\}$ basis for $D^{\prime}$ with $E$ the Euler derivation and $\operatorname{deg}\left(\theta_{1}\right)=m, \operatorname{deg}\left(\theta_{2}\right)=a$. We now use that $D \subset D^{\prime}$. 
- $m<a$. Since $\theta \in D_{0}, \theta=f E+c \theta_{1}$ for some $c \in \mathbb{C}^{*}$. Then $\left\{E, \theta, \theta_{2}\right\}$ is a basis for $D^{\prime}$, so by Saito's criterion $\left\{E, \theta, C \theta_{2}\right\}$ is a basis for $D$.

- $m=a$. Then $\theta=f E+c_{1} \theta_{1}+c_{2} \theta_{2}$, where $c_{1}, c_{2}$ constants, not both zero. If $c_{2} \neq 0$, then $\left\{E, \theta_{1}, \theta\right\}$ is a basis for $D^{\prime}$, so by Saito's criterion $\left\{E, C \theta_{1}, \theta\right\}$ is a basis for $D$.

- $m=a+1$. Then $\theta=f E+c_{1} \theta_{1}+L_{2} \theta_{2}$, where $c$ is a constant and $L_{2}$ is a linear form, not both zero. If $c_{1}=0$ then $L_{2} \theta_{2}(C) \in\langle C\rangle$. Since $C$ is irreducible, then $\theta_{2}(C) \in\langle C\rangle$, and so $\theta_{2} \in D_{0}$ is of degree $a<m, a+2$. This is inconsistent with the Hilbert series of $D_{0}$. So $c_{1} \neq 0$, and so $\left\{E, \theta, \theta_{2}\right\}$ is a basis for $D^{\prime}$, and again by Saito's criterion $\left\{E, \theta, C \theta_{2}\right\}$ is a basis for $D$.

- $m=a+2$. Then $\theta=f_{1} E+c_{1} \theta_{1}+g_{1} \theta_{2}$, where $c_{1}$ is a constant and $g_{1}$ is a quadratic form, not both zero and $\eta=f_{2} E+c_{2} \theta_{1}+g_{2} \theta_{2}$, where $c_{2}$ is a constant and $g_{2}$ is a quadratic form, not both zero. If $c_{1}=c_{2}=0$, then either $g_{i}=c_{i}^{\prime} C, c_{i}^{\prime} \neq 0, i=1,2$ or $\theta_{2} \in D_{0}$, a contradiction (because $\operatorname{deg}\left(\theta_{2}\right)=a$ ). Therefore $c_{2}^{\prime} \theta-c_{1}^{\prime} \eta \in D_{0} \cap E S=\{0\}$, contradicting the fact that $\theta, \eta$ are minimal generators of $D_{0}$. So if $c_{2} \neq 0$, then $\left\{E, \eta, \theta_{2}\right\}$ is a basis for $D^{\prime}$, and so by Saito's criterion $\left\{E, \eta, C \theta_{2}\right\}$ is a basis for $D$.

- $m>a+2$. Then $\theta=f_{1} E+c_{1} \theta_{1}+g_{1} \theta_{2}$, where $c_{1}$ is a constant and $g_{1}$ is a polynomial, not both zero and $\eta=f_{2} E+c_{2} \theta_{1}+g_{2} \theta_{2}$, where $c_{2}$ is a constant and $g_{2}$ is a quadratic form, not both zero. If $c_{1}=c_{2}=0$, then $g_{1}=C g_{1}^{\prime}, g_{1}^{\prime} \neq 0$ and $g_{2}=c_{2}^{\prime} C, c_{2}^{\prime}$ nonzero constant, and the argument used above yields a contradiction. So $c_{1} \neq 0$ or $c_{2} \neq 0$. Applying Saito's criterion yields the desired result.

Case 2: $k=2 m+1, m=a$. By Lemma 3.8,

$$
H S\left(D_{0}, t\right)=\frac{2 t^{m+1}}{(1-t)^{3}}
$$

This implies there exist degree $m+1$ minimal generators $\theta, \mu \in D_{0}$. Suppose $\left\{E, \theta_{1}, \theta_{2}\right\}$ is a basis for $D^{\prime}$ where $E$ is the Euler derivation and $\operatorname{deg}\left(\theta_{1}\right)=m$, $\operatorname{deg}\left(\theta_{2}\right)=m$. So $\theta=f_{1} E+L_{1} \theta_{1}+K_{1} \theta_{2}$ and $\mu=f_{2} E+L_{2} \theta_{1}+K_{2} \theta_{2}$, where $L_{1}, L_{2}, K_{1}, K_{2}$ are linear forms, and for any $i=1,2, L_{i}, K_{i}$ cannot be simultaneously zero. Hence $L_{2} \theta-L_{1} \mu-\left(L_{2} f_{1}-L_{1} f_{2}\right) E=\left(L_{2} K_{1}-L_{1} K_{2}\right) \theta_{2} \in D(\mathcal{C})$. But $\theta_{2}$ is in $D\left(C^{\prime}\right)$ and $\theta_{2}(C) \notin\langle C\rangle$, else $\left(D_{0}\right)_{m}$ is nonzero, which is inconsistent with the Hilbert series. Hence $L_{2} K_{1}-L_{1} K_{2}=c C$, where $c$ is a constant.

If $c=0$, then $L_{1}=u K_{1}, L_{2}=u K_{2}, u \neq 0$ or $L_{1}=v L_{2}, K_{1}=v K_{2}, v \neq 0$, where $u, v$ are constants, and that $K_{2} f_{1}=K_{1} f_{2}$ and $L_{2} f_{1}=L_{1} f_{2}$. If $L_{1}=u K_{1}$, $L_{2}=u K_{2}, u \neq 0$, and $K_{1} \neq c t \cdot K_{2}$ we get $\theta=K_{1}\left(g E+u \theta_{1}+\theta_{2}\right)$. Since $K_{1} \neq 0$ (else $L_{1}=0$ ) then $\theta(C) \in\langle C\rangle$ implies $\left(g E+u \theta_{1}+\theta_{2}\right)(C) \in\langle C\rangle$, yielding a degree $m$ derivation in $D(\mathcal{C})$, a contradiction. If $K_{1}=c t \cdot K_{2}$, then $\theta$ 
and $\mu$ are not minimal generators, also a contradiction. If $c \neq 0$, then we find $\operatorname{det}[E, \theta, \mu]=c C \operatorname{det}\left[E, \theta_{1}, \theta_{2}\right]$, and Saito's criterion shows that $\{E, \theta, \mu\}$ is a basis for $D(\mathcal{C})$.

Case 3: $k=2 m+1, m \neq a$. By Lemma 3.8,

$$
H S\left(D_{0}, t\right)=\frac{t^{a+2}+2 t^{m+1}-t^{m+2}}{(1-t)^{3}} .
$$

Since $m \neq a$, there is no cancellation in the numerator, hence $D_{0}$ cannot be free.

Lemma 3.10. Let $\left(\mathcal{C}^{\prime}, \mathcal{C}, \mathcal{C}^{\prime \prime}\right)$ be a quasihomogeneous triple, with $\left|C \cap \mathcal{C}^{\prime}\right|=$ $\left|\mathcal{C}^{\prime \prime}\right|=k$. If $\mathcal{C}$ is free, then

(1) If $k=2 m$ and $\exp (\mathcal{C})=\{1, m, a+2\}$, then $\mathcal{C}^{\prime}$ is free with $\exp \left(\mathcal{C}^{\prime}\right)=\{1, m, a\}$.

(2) If $k=2 m+1$ and $\exp (\mathcal{C})=\{1, m+1, m+1\}$, then $\mathcal{C}^{\prime}$ is free with $\exp \left(\mathcal{C}^{\prime}\right)=$ $\{1, m, m\}$.

(3) If $k=2 m+1$ and $\exp (\mathcal{C})=\{1, m+1, a+1\}$ with $a \neq m$, then $\complement^{\prime}$ is not free.

Proof. As in Lemma 2.13, apply $\operatorname{Hom}\left(-, \mathcal{O}_{\mathbb{P}^{2}}\right)$ to the exact sequence

$$
0 \longrightarrow \mathscr{D}_{0}^{\prime}(-2) \stackrel{\cdot C}{\longrightarrow} \mathscr{D}_{0} \longrightarrow i_{*} \mathcal{O}_{L}(-k) \longrightarrow 0 .
$$

Since $i_{*} \mathcal{O}_{L}(-k)$ is supported on the conic $C, \operatorname{Hom}\left(i_{*} \mathcal{O}_{L}(-k), \mathcal{O}_{\mathbb{P}^{2}}\right)=0$. The assumption that $D_{0}$ is free implies that $\operatorname{Ext}_{S}^{1}\left(\mathscr{D}_{0}, \mathcal{O}_{\mathbb{P}^{2}}\right)=0$. This yields an exact sequence:

$$
0 \longrightarrow \mathscr{D}_{0}^{\vee} \longrightarrow \mathscr{D}_{0}^{\prime \vee}(2) \longrightarrow \operatorname{Ext}_{S}^{1}\left(i_{*} \mathcal{O}_{L}(-k), \mathcal{O}_{\mathbb{P}^{2}}\right) \longrightarrow 0 .
$$

As $D_{0}$ free with known exponents, so also is $D_{0}^{\vee}$, and the Hilbert series is known. The proof of Lemma 3.6 provides a free resolution of $i_{*} d_{E}$, which allows us to compute $\operatorname{Ext}_{S}^{1}\left(i_{*} d_{E}, S\right)$. Combining everything yields the Hilbert series of $D_{0}^{\prime \vee}$, and the result follows as in the previous analysis.

\section{Freeness of $C L$ arrangements is not combinatorial}

We close with a pair of examples which show that in the CL case, Terao's conjecture that freeness is a combinatorial invariant of an arrangement is false.

Example 4.1. Let $\ell_{1}$ be given by

$$
\begin{aligned}
& C_{1}=\left\{y^{2}+x z=0\right\}, \\
& C_{2}=\left\{y^{2}+x^{2}+2 x z=0\right\}, \\
& L_{1}=\{x=0\}
\end{aligned}
$$


$L_{1}$ is tangent to both $C_{1}$ and $C_{2}$ at the point $P=(0: 0: 1) ; C_{1}$ and $C_{2}$ have two other points in common. Adding the line $L=\{y=0\}$ passing through $P$ to $C_{1}$ yields a quasihomogeneous, free $C L$ arrangement $\mathcal{C}$, with $D_{0}(\mathcal{C}) \simeq S(-2) \oplus S(-3)$ :

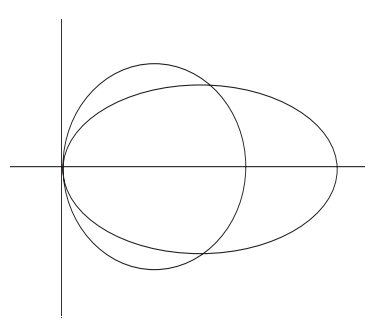

The line $L^{\prime}=\{x-13 y=0\}$ passes through $P$, and misses the other singularities of $\ell_{1}$. The $\mathrm{CL}$ arrangement $\ell^{\prime}=L^{\prime} \cup \mathcal{C}_{1}$ is combinatorially identical to $\mathcal{C}$, but $\ell^{\prime}$ is not quasihomogeneous, and not free:

$$
0 \longrightarrow S(-9) \longrightarrow S(-8)^{3} \longrightarrow D_{0}\left(e^{\prime}\right) \longrightarrow 0 .
$$

Even for CL arrangements with ordinary singularities, there are counterexamples:

Example 4.2. Let $\mathcal{A}$ be the union of the five smooth conics:

$$
\begin{aligned}
& C_{1}=(x-3 z)^{2}+(y-4 z)^{2}-25 z^{2}=0, \\
& C_{2}=(x-4 z)^{2}+(y-3 z)^{2}-25 z^{2}=0, \\
& C_{3}=(x+3 z)^{2}+(y-4 z)^{2}-25 z^{2}=0, \\
& C_{4}=(x+4 z)^{2}+(y-3 z)^{2}-25 z^{2}=0, \\
& C_{5}=(x-5 z)^{2}+y^{2}-25 z^{2}=0 .
\end{aligned}
$$

$\mathcal{A}$ has 13 singular points, all ordinary. At 10 of these points only two branches of $\mathcal{A}$ meet, while at the points $(0: 0: 1),(1: i: 0),(1:-i: 0)$, all five conics meet. The Milnor and Tjurina numbers agree at all singularities except $(0: 0: 1)$, where $\tau=15$ and $\mu=16$. Adding lines $L_{1}, L_{2}, L_{3}$ connecting $(0: 0: 1),(1: i: 0),(1:-i: 0)$

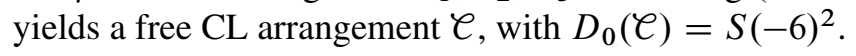

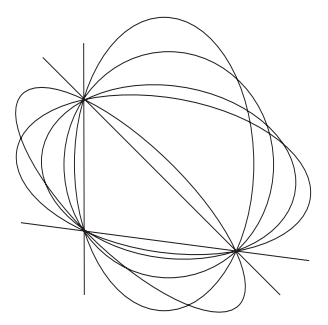


Next, let $\mathcal{A}^{\prime}$ be the union of the following five smooth conics:

$$
\begin{aligned}
& C_{1}=x^{2}+8 y^{2}+21 x y-x z-8 y z=0, \\
& C_{2}=x^{2}+5 y^{2}+13 x y-x z-5 y z=0, \\
& C_{3}=x^{2}+9 y^{2}-4 x y-x z-9 y z=0, \\
& C_{4}=x^{2}+11 y^{2}+x y-x z-11 y z=0, \\
& C_{5}=x^{2}+17 y^{2}-5 x y-x z-17 y z=0 .
\end{aligned}
$$

$\mathcal{A}^{\prime}$ is combinatorially identical to $\mathcal{A}$, but at the points $(0: 0: 1),(1: 0: 1),(0: 1: 1)$ where all the branches meet, $\tau=15$ and $\mu=16$. Adding the lines connecting these three points yields a CL arrangement $\ell^{\prime}$ which is combinatorially identical to $\ell$ but not free; the free resolution of $D_{0}\left(\mathcal{C}^{\prime}\right)$ is:

$$
0 \longrightarrow S(-8)^{2} \longrightarrow S(-7)^{4} \longrightarrow D_{0}\left(\ell^{\prime}\right) \longrightarrow 0 .
$$

As was pointed out by the referee, the complements of arrangements $\mathcal{A}$ and $\mathcal{A}^{\prime}$ are homeomorphic (via a Cremona transformation centered on the three multiple intersection points) to the complements of a pair of line arrangements consisting of eight lines in general position. The moduli space of such objects is connected, so the complements are rigidly isotopic, hence homeomorphic. So freeness is also not a topological invariant.

\section{Concluding remarks}

(1) As noted in $\S 1.2$, for the complement $X$ of a CL arrangement in $\mathbb{P}^{2}$ the Betti numbers $h^{1}(X)$ and $h^{2}(X)$ depend only on the combinatorics, and so if $X$ is quasihomogeneous and free, there is a version of Terao's theorem, which we leave for the interested reader.

(2) In the examples above, the Jacobian ideals are of different degrees, so are not even members of the same Hilbert scheme. Do there exist CL arrangements with isomorphic intersection poset and singularities which are locally isomorphic, one free and one nonfree? Do there exist counterexamples where all singularities are quasihomogeneous?

(3) As shown by Example 2.3, quasihomogenity is not a necessary condition for freeness of CL arrangements. However, without this assumption, the sequences in Propositions 2.8 and 3.8 may not be exact, which means that any form of addition-deletion will require hypotheses on higher cohomology.

Acknowledgments. Macaulay2 computations were essential to our work. We also thank an anonymous referee for many useful suggestions, in particular for pointing out that we should remove one of our original conditions (that $\mathcal{C}$ has only ordinary singularities). 


\section{References}

[1] T. Abe, H. Terao, M. Wakefield, The Euler multiplicity and addition-deletion theorems for multiarrangements. J. London Math. Soc. 77 (2008), 335-348. Zbl 1140.52015 MR 2400395

[2] J. I. Cogolludo-Agustín, Topological invariants of the complement to arrangements of rational plane curves. Mem. Amer. Math. Soc. 159 (2002), no. 756. Zbl 1038.32025 MR 1921584

[3] D. Eisenbud, Commutative Algebra with a view towards Algebraic Geometry. Grad. Texts in Math. 150, Springer-Verlag, New York 1995. Zbl 0819.13001 MR 1322960

[4] M. Mustaţă, H. Schenck, The module of logarithmic p-forms of a locally free arrangement. J. Algebra 241 (2001), 699-719. Zbl 1047.14007 MR 1843320

[5] P. Orlik, H. Terao, Arrangements of hyperplanes. Grundlehren Math. Wiss. 300, SpringerVerlag, Berlin 1992. Zbl 0757.55001 MR 1217488

[6] H. Reiffen, Das Lemma von Poincaré für holomorphe Differentialformen auf komplexen Räumen. Math. Z. 101 (1967), 269-284. Zbl 0164.09401 MR 0223599

[7] K. Saito, Theory of logarithmic differential forms and logarithmic vector fields. J. Fac. Sci. Univ. Tokyo Sect. IA Math. 27 (1980), 265-291. Zbl 0496.32007 MR 0586450

[8] K. Saito, Quasihomogene isolierte Singularitäten von Hyperflächen. Invent. Math. 14 (1971), 123-142. Zbl 0224.32011 MR 0294699

[9] H. Schenck, A rank two vector bundle associated to a three arrangement, and its Chern polynomial. Adv. Math. 149 (2000), 214-229. Zbl 0977.52029 MR 1742707

[10] H. Schenck, Elementary modifications and line configurations in $\mathbb{P}^{2}$. Comment. Math. Helv. 78 (2003), 447-462. Zbl 1033.52018 MR 1998388

[11] H. Terao, Generalized exponents of a free arrangement of hyperplanes and Shepard-ToddBrieskorn formula. Invent. Math. 63 (1981), 159-179. Zbl 0437.51002 MR MR0608532

[12] H. Terao, Arrangements of hyperplanes and their freeness I. J. Fac. Sci. Univ. Tokyo 27 (1980), 293-312. Zbl 0509.14006 MR 0586451

[13] M. Wakefield, S. Yuzvinsky, Derivations of an effective divisor on the complex projective line. Trans. Amer. Math. Soc. 359 (2007), 4389-4403. Zbl 1121.52036 MR 2309190

[14] C. T. C. Wall, Singular points of plane curves. Cambridge University Press, Cambridge 2004. Zbl 1057.14001 MR 2107253

[15] G. Ziegler, Multiarrangements of hyperplanes and their freeness. In Singularities, Contemp. Math. 90, Amer. Math. Soc., Providence, RI, 1989, 345-359. Zbl 0678.51010 MR 1000610

[16] M. Yoshinaga, Characterization of a free arrangement and conjecture of Edelman and Reiner. Invent. Math. 157 (2004), 449-454. Zbl 1113.52039 MR 2077250

[17] M. Yoshinaga, On the freeness of 3-arrangements. Bull. London Math. Soc. 37 (2005), 126-134. Zbl 1071.52019 MR 2105827

Received March 27, 2007 
Hal Schenck, Mathematics Department, Texas A\&M University, College Station,

TX 77843-3368, and Mathematics Department, University of Illinois, Urbana, IL 61801, U.S.A.

E-mail: schenck@math.uiuc.edu

Ştefan O. Tohăneanu, Mathematics Department, Texas A\&M University, College Station,

TX 77843-3368, and Department of Mathematical Sciences, University of Cincinnati, Cincinnati, OH 45221-0025, U.S.A.

E-mail: stefan.tohaneanu@uc.edu 